PROCEEDINGS OF THE

AMERICAN MATHEMATICAL SOCIETY

Volume 138, Number 1, January 2010, Pages 287-297

S 0002-9939(09)10061-8

Article electronically published on August 25, 2009

\title{
BOUNDED APPROXIMATION PROPERTIES VIA INTEGRAL AND NUCLEAR OPERATORS
}

\author{
ÅSVALD LIMA, VEGARD LIMA, AND EVE OJA \\ (Communicated by Nigel J. Kalton)
}

\begin{abstract}
Let $X$ be a Banach space and let $\mathcal{A}$ be a Banach operator ideal. We say that $X$ has the $\lambda$-bounded approximation property for $\mathcal{A}(\lambda$-BAP for $\mathcal{A})$ if for every Banach space $Y$ and every operator $T \in \mathcal{A}(X, Y)$, there exists a net $\left(S_{\alpha}\right)$ of finite rank operators on $X$ such that $S_{\alpha} \rightarrow I_{X}$ uniformly on compact subsets of $X$ and

$$
\limsup _{\alpha}\left\|T S_{\alpha}\right\|_{\mathcal{A}} \leq \lambda\|T\|_{\mathcal{A}}
$$

We prove that the (classical) $\lambda$-BAP is precisely the $\lambda$-BAP for the ideal $\mathcal{I}$ of integral operators, or equivalently, for the ideal $\mathcal{S I}$ of strictly integral operators. We also prove that the weak $\lambda$-BAP is precisely the $\lambda$-BAP for the ideal $\mathcal{N}$ of nuclear operators.
\end{abstract}

\section{INTRODUCTION}

Let $X$ and $Y$ be Banach spaces. We denote by $\mathcal{L}(X, Y)$ the Banach space of all bounded linear operators from $X$ to $Y$, and by $\mathcal{F}(X, Y)$ and $\mathcal{W}(X, Y)$ its subspaces of finite rank and weakly compact operators. Let $I_{X}$ denote the identity operator on $X$. Recall that $X$ is said to have the approximation property (AP) if there exists a net $\left(S_{\alpha}\right) \subset \mathcal{F}(X, X)$ such that $S_{\alpha} \rightarrow I_{X}$ uniformly on compact subsets of $X$. If $\left(S_{\alpha}\right)$ can be chosen with $\sup _{\alpha}\left\|S_{\alpha}\right\| \leq \lambda$ for some $\lambda \geq 1$, then $X$ is said to have the $\lambda$-bounded approximation property ( $\lambda$-BAP).

Recently, the weak bounded approximation property was introduced and studied in [11. We say that $X$ has the weak $\lambda$-bounded approximation property (weak $\lambda$ BAP) if for every Banach space $Y$ and every operator $T \in \mathcal{W}(X, Y)$ there exists a net $\left(S_{\alpha}\right) \subset \mathcal{F}(X, X)$ such that $S_{\alpha} \rightarrow I_{X}$ uniformly on compact subsets of $X$ and $\lim \sup _{\alpha}\left\|T S_{\alpha}\right\| \leq \lambda\|T\|$. Thus the weak BAP can be characterized as the AP which is bounded for every weakly compact operator. This suggests the following definition.

Let $X$ be a Banach space and let $\mathcal{A}=\left(\mathcal{A},\|\|_{\mathcal{A}}\right)$ be a Banach operator ideal. We say that $X$ has the $\lambda$-bounded approximation property for $\mathcal{A}(\lambda$-BAP for $\mathcal{A})$

Received by the editors April 17, 2009, and, in revised form, May 29, 2009.

2000 Mathematics Subject Classification. Primary 46B28; Secondary 46B20, 47B10, 47L05, $47 \mathrm{~L} 20$.

Key words and phrases. Banach spaces, Banach operator ideals, bounded approximation properties.

The research of the third author was partially supported by Estonian Science Foundation Grant No. 7308 .

(C)2009 American Mathematical Society Reverts to public domain 28 years from publication 
if for every Banach space $Y$ and every operator $T \in \mathcal{A}(X, Y)$ there exists a net $\left(S_{\alpha}\right) \subset \mathcal{F}(X, X)$ such that $S_{\alpha} \rightarrow I_{X}$ uniformly on compact subsets of $X$ and

$$
\underset{\alpha}{\limsup }\left\|T S_{\alpha}\right\|_{\mathcal{A}} \leq \lambda\|T\|_{\mathcal{A}}
$$

The weak $\lambda$-BAP is, by definition, the $\lambda$-BAP for the ideal $\mathcal{W}$ of weakly compact operators. In [11] it was proved that the weak $\lambda$-BAP is also equivalent to the $\lambda$ $\mathrm{BAP}$ for the ideal $\mathcal{K}$ of compact operators. It is immediate that the $\lambda$-BAP implies the $\lambda$-BAP for every Banach operator ideal $\mathcal{A}$ (since $\left\|T S_{\alpha}\right\|_{\mathcal{A}} \leq\|T\|_{\mathcal{A}}\left\|S_{\alpha}\right\|$ ), and it is equivalent to the $\lambda$-BAP for the ideal $\mathcal{L}$ of all operators.

Since $\mathcal{L}$ is the largest of all Banach operator ideals, it is natural to ask whether there is some kind of smallest Banach operator ideal $\mathcal{A}$ for which the BAP for $\mathcal{A}$ would be equivalent to the BAP. Concerning the weak BAP, $\mathcal{K}$ is already a quite "small" Banach operator ideal. But what about the smallest Banach operator ideal $\mathcal{A}$ for which the BAP for $\mathcal{A}$ would be equivalent to the weak BAP?

Our main results (see Sections 2 and 3, respectively) are that (1) the BAP is precisely the BAP for the ideal $\mathcal{I}$ of integral operators or, equivalently, for the ideal $\mathcal{S I}$ of strictly integral operators (Pietsch integral, in [5] and [22]); and (2) the weak $\mathrm{BAP}$ is precisely the BAP for the ideal $\mathcal{N}$ of nuclear operators. Notice that $\mathcal{N}$ is the smallest of all Banach operator ideals and $\mathcal{S I}$ is considered to be the continuous analogue of $\mathcal{N}$. Moreover, $\mathcal{N} \subset \mathcal{S I} \subset \mathcal{I}$, and $\mathcal{I}$ is known to be the smallest maximal Banach operator ideal. We also indicate (see Section 4) a particular role played by $\ell_{1}$ and $\ell_{1}^{* *}$ in the case of the weak BAP and the BAP, respectively.

To complete the picture, let us recall other types of bounded approximation properties involving operator ideals which have been studied since the early $1980 \mathrm{~s}$ (see, e.g., 14 for references). Let $\mathcal{A}$ be an operator ideal. A Banach space $X$ is said to have the $\lambda$-bounded $\mathcal{A}$-approximation property ( $\lambda$-bounded $\mathcal{A}$-AP) if there exists a net $\left(S_{\alpha}\right) \subset \mathcal{A}(X, X)$ with $\sup _{\alpha}\left\|S_{\alpha}\right\| \leq \lambda$ such that $S_{\alpha} \rightarrow I_{X}$ uniformly on compact subsets of $X$. Here the operator norm \|\| is used for all $\mathcal{A}$, and not the operator ideal norm \|\|$_{\mathcal{A}}$. Otherwise, the notion would be very restrictive; e.g., even $\ell_{2}$ would not have the bounded $\mathcal{N}$-AP (see [16, Remark 2.1]). Our notion of the BAP for $\mathcal{A}$ seems to be the first attempt to define an AP related to a Banach operator ideal $\mathcal{A}$ that also depends on the operator ideal norm of $\mathcal{A}$, and not only on the operator ideal properties of $\mathcal{A}$.

Our notation is standard. A Banach space $X$ will be regarded as a subspace of its bidual $X^{* *}$ under the canonical embedding $j_{X}: X \rightarrow X^{* *}$. The closure of a set $A \subset X$ is denoted $\bar{A}$. The tensor product $X \otimes Y$ with a tensor norm $\alpha$ is denoted by $X \otimes_{\alpha} Y$ and its completion by $X \hat{\otimes}_{\alpha} Y$. We shall use only the classical projective tensor norm $\pi=\|\|_{\pi}$ and the injective tensor norm $\varepsilon$. Since $\mathcal{F}(X, Y)=X^{*} \otimes Y$, we shall write $\|T\|_{\pi}$ for $T \in \mathcal{F}(X, Y)\left(\|\|_{\pi}\right.$ is called the finite nuclear norm in [20]). Let us recall that for Banach operator ideals $\mathcal{A}$ and $\mathcal{B}$ the inclusion $\mathcal{A} \subset \mathcal{B}$ means that $\mathcal{A}(X, Y) \subset \mathcal{B}(X, Y)$ and $\|T\|_{\mathcal{A}} \geq\|T\|_{\mathcal{B}}$ for all Banach spaces $X$ and $Y$ and for all operators $T \in \mathcal{A}(X, Y)$.

We refer to the books by Diestel-Uhl [5] and Ryan [22] for the classical approximation properties, tensor products, and for the common Banach operator ideals such as $\mathcal{N}, \mathcal{S I}$, and $\mathcal{I}$; see also [4] by Diestel, Jarchow, and Tonge, and Pietsch's book 20] for operator ideals. Following [4, we use the term strictly integral operator for Pietsch integral operator in [5] and [22]. And we use Banach operator ideal 
for normed operator ideal in 20, or for Banach ideal in [4] and 22] (note that, in the Banach spaces context, the term ideal has its own meaning (see Section 4)).

\section{The Bounded APPROXIMATION PROPERTY VIA INTEGRAL OPERATORS}

We shall establish the following reformulations of the BAP in terms of the boundedness for the Banach operator ideals $\mathcal{S I}$ and $\mathcal{I}$ of strictly integral and integral operators, respectively.

Theorem 2.1. Let $X$ be a Banach space, and let $1 \leq \lambda<\infty$. The following statements are equivalent.

(a) $X$ has the $\lambda-B A P$.

(b) $X$ has the $\lambda$-BAP for $\mathcal{S I}$.

(c) $X$ has the $\lambda$-BAP for $\mathcal{I}$.

We shall need the well-known reformulation of the BAP (cf., e.g., [3, p. 193] or [22, p. 80]) which is essentially due to Grothendieck [7.

Theorem 2.2 (Grothendieck). Let $X$ be a Banach space, and let $1 \leq \lambda<\infty$. The following statements are equivalent.

(a) $X$ has the $\lambda$-BAP.

(b) $\|T\|_{\pi} \leq \lambda\|T\|_{\mathcal{I}}$ for all $T \in \mathcal{F}(X, X)$.

Lemma 2.3. Let $X$ be a Banach space, and let $1 \leq \lambda<\infty$. If a Banach space $Y$ has the property that for every $T \in \mathcal{I}\left(X, Y^{* *}\right)$ there exists a net $\left(S_{\alpha}\right) \subset \mathcal{F}(X, X)$ such that $S_{\alpha} \rightarrow I_{X}$ pointwise and $\lim \sup _{\alpha}\left\|T S_{\alpha}\right\|_{\pi} \leq \lambda\|T\|_{\mathcal{I}}$, then every quotient space of $Y$ has the same property.

Proof. Denote by $q: Y \rightarrow Z$ the quotient mapping, and let $U \in \mathcal{I}\left(X, Z^{* *}\right)$. We are going to use well-known facts about tensor products (see, e.g., [5] or 22]). Since $\mathcal{I}\left(X, Z^{* *}\right)=\left(Z^{*} \hat{\otimes}_{\varepsilon} X\right)^{*}$ and $Z^{*} \hat{\otimes}_{\varepsilon} X$ is a subspace of $Y^{*} \hat{\otimes}_{\varepsilon} X$ (under the isometric embedding $q^{*} \otimes I_{X}$; notice that $q^{*}$ is an isometric embedding), we may consider a norm-preserving extension of $U$. Thus, there exists $T \in\left(Y^{*} \hat{\otimes}_{\varepsilon} X\right)^{*}=\mathcal{I}\left(X, Y^{* *}\right)$, such that $\|T\|_{\mathcal{I}}=\|U\|_{\mathcal{I}}$ and

$$
(U x)\left(z^{*}\right)=\left\langle U, z^{*} \otimes x\right\rangle=\left\langle T, q^{*} z^{*} \otimes x\right\rangle=(T x)\left(q^{*} z^{*}\right)=\left(q^{* *} T x\right)\left(z^{*}\right)
$$

for all $x \in X$ and $z^{*} \in Z^{*}$, meaning that $U=q^{* *} T$.

Let $S \in \mathcal{F}(X, X)$. Then $U S \in \mathcal{F}\left(X, Z^{* *}\right)=X^{*} \otimes Z^{* *}$ and

$$
\|U S\|_{\pi}=\left\|q^{* *} T S\right\|_{\pi}=\left\|\left(I_{X^{*}} \otimes q^{* *}\right)(T S)\right\|_{\pi} \leq\|T S\|_{\pi} .
$$

Now if $\left(S_{\alpha}\right) \subset \mathcal{F}(X, X)$ is chosen for $T$, then we also have

$$
\limsup _{\alpha}\left\|U S_{\alpha}\right\|_{\pi} \leq \limsup _{\alpha}\left\|T S_{\alpha}\right\|_{\pi} \leq \lambda\|T\|_{\mathcal{I}}=\lambda\|U\|_{\mathcal{I}}
$$

as needed.

Lemma 2.4. Let $X$ be a Banach space, and let $T \in \mathcal{F}(X, X)=X^{*} \otimes X$. Then there exist $A \in \mathcal{L}\left(X^{*}, X^{*}\right)$ with $\|A\|=1$ and $V \in \mathcal{F}(X, X)$ such that $V^{*}=A T^{*}$ and

$$
\|T\|_{\pi} \leq \limsup _{\alpha}\left\|j_{X} V S_{\alpha}\right\|_{\pi}
$$

for every net $\left(S_{\alpha}\right) \subset \mathcal{F}(X, X)$ converging pointwise to the identity $I_{X}$. 
Proof. Let $T=\sum_{n=1}^{m} x_{n}^{*} \otimes x_{n} \in X^{*} \otimes X$. Using the canonical description $\left(X^{*} \hat{\otimes}_{\pi} X\right)^{*}$ $=\mathcal{L}\left(X^{*}, X^{*}\right)$, we find $A \in \mathcal{L}\left(X^{*}, X^{*}\right)$ with $\|A\|=1$ such that

$$
\|T\|_{\pi}=\sum_{n=1}^{m}\left(A x_{n}^{*}\right)\left(x_{n}\right)=\operatorname{trace}(V)
$$

where

$$
V=\sum_{n=1}^{m} A x_{n}^{*} \otimes x_{n} \in \mathcal{F}(X, X) .
$$

It is easily verified that $V^{*}=A T^{*}$.

Let $\left(S_{\alpha}\right) \subset \mathcal{F}(X, X)$ be a net such that $S_{\alpha} \rightarrow I_{X}$ pointwise. Since $X^{*} \hat{\otimes}_{\pi} X$ is a subspace of $X^{*} \hat{\otimes}_{\pi} X^{* *}$ (under the canonical inclusion $I_{X^{*}} \otimes j_{X}$ ), for all $\alpha$, we have

$$
\left\|V S_{\alpha}\right\|_{\pi}=\left\|j_{X} V S_{\alpha}\right\|_{\pi} .
$$

Therefore,

$$
\begin{aligned}
& \|T\|_{\pi}=\sum_{n=1}^{m}\left(A x_{n}^{*}\right)\left(x_{n}\right)=\lim _{\alpha} \sum_{n=1}^{m}\left(A x_{n}^{*}\right)\left(S_{\alpha} x_{n}\right)=\lim _{\alpha} \sum_{n=1}^{m}\left(S_{\alpha}^{*} A x_{n}^{*}\right)\left(x_{n}\right) \\
& =\lim _{\alpha} \operatorname{trace}\left(V S_{\alpha}\right) \leq \limsup _{\alpha}\left\|V S_{\alpha}\right\|_{\pi}=\limsup _{\alpha}\left\|j_{X} V S_{\alpha}\right\|_{\pi}
\end{aligned}
$$

as desired.

Proof of Theorem 2.1. The implications $(\mathrm{a}) \Rightarrow(\mathrm{b})$ and $(\mathrm{a}) \Rightarrow(\mathrm{c})$ are obvious as noted in the Introduction.

If (b) or (c) holds, then for every $\ell_{1}(\Gamma)$-space and for every $T \in \mathcal{I}\left(X, \ell_{1}(\Gamma)^{* *}\right)=$ $\mathcal{S I}\left(X, \ell_{1}(\Gamma)^{* *}\right)$ there exists a net $\left(S_{\alpha}\right) \subset \mathcal{F}(X, X)$ such that $S_{\alpha} \rightarrow I_{X}$ pointwise and $\lim \sup _{\alpha}\left\|T S_{\alpha}\right\|_{\mathcal{I}} \leq \lambda\|T\|_{\mathcal{I}}$. Since $T S_{\alpha} \in \mathcal{F}\left(X, \ell_{1}(\Gamma)^{* *}\right)=X^{*} \otimes \ell_{1}(\Gamma)^{* *}$ and $\ell_{1}(\Gamma)^{* *}$ has the metric AP, it is well known (see, e.g., [22, p. 176]) that

$$
\left\|T S_{\alpha}\right\|_{\pi}=\left\|T S_{\alpha}\right\|_{\mathcal{N}}=\left\|T S_{\alpha}\right\|_{\mathcal{I}} .
$$

Therefore, recalling that every Banach space is a quotient of some $\ell_{1}(\Gamma)$-space and applying Lemma 2.3 we may assume that for every $U \in \mathcal{I}\left(X, X^{* *}\right)$ there exists $\left(S_{\alpha}\right)$ as above such that

$$
\limsup _{\alpha}\left\|U S_{\alpha}\right\|_{\pi} \leq \lambda\|U\|_{\mathcal{I}} .
$$

Let $T \in \mathcal{F}(X, X)$. Choose $A$ and $V$ as in Lemma 2.4. Then choose a net $\left(S_{\alpha}\right) \subset \mathcal{F}(X, X)$ to be pointwise convergent to $I_{X}$ such that

$$
\limsup \left\|j_{X} V S_{\alpha}\right\|_{\pi} \leq \lambda\left\|j_{X} V\right\|_{\mathcal{I}} .
$$

Then, by Lemma 2.4

$$
\|T\|_{\pi} \leq \lambda\left\|j_{X} V\right\|_{\mathcal{I}}=\lambda\|V\|_{\mathcal{I}}
$$

On the other hand, since $V^{*}=A T^{*}$,

$$
\|V\|_{\mathcal{I}}=\left\|V^{*}\right\|_{\mathcal{I}}=\left\|A T^{*}\right\|_{\mathcal{I}} \leq\left\|T^{*}\right\|_{\mathcal{I}}=\|T\|_{\mathcal{I}} .
$$

In conclusion,

$$
\|T\|_{\pi} \leq \lambda\|T\|_{\mathcal{I}},
$$

which means, according to Theorem 2.2, that $X$ has the $\lambda$-BAP. 


\section{ThE WEAK BOUNDED APPROXIMATION PROPERTY VIA NUCLEAR OPERATORS}

The smallest Banach operator ideal $\mathcal{N}$ of nuclear operators is known to be the discrete analogue of $\mathcal{S I}$ (see, e.g., [4, pp. 111-113]). For the weak BAP, $\mathcal{N}$ plays the same role as $\mathcal{S I}$ for the BAP.

Theorem 3.1. Let $X$ be a Banach space, and let $1 \leq \lambda<\infty$. The following statements are equivalent.

(a) $X$ has the weak $\lambda-B A P$.

(b) $X$ has the $\lambda$-BAP for $\mathcal{N}$.

The proof of Theorem 3.1 will rely on the following recent reformulation of the weak BAP (which is to be compared with Theorem 2.2).

Theorem 3.2 (see [19, Theorem 3.6]). Let $X$ be a Banach space, and let $1 \leq \lambda<$ $\infty$. The following statements are equivalent.

(a) $X$ has the weak $\lambda$-BAP.

(b) $\|T\|_{\pi} \leq \lambda\left\|j_{X} T\right\|_{\mathcal{N}}$ for all $T \in \mathcal{F}(X, X)$.

The proof of Theorem 3.1 will also use a reformulation of the weak BAP in terms of extension operators. Let $X$ be a closed subspace of a Banach space $W$. An operator $\Phi \in \mathcal{L}\left(X^{*}, W^{*}\right)$ is called an extension operator if $\left(\Phi x^{*}\right)(x)=x^{*}(x)$ for all $x^{*} \in X^{*}$ and all $x \in X$.

Theorem 3.3 (see [12, Propositions 2.1, 2.3, and 2.5] and [19, Corollary 3.18]). Let $X$ be a Banach space, and let $1 \leq \lambda<\infty$. The following statements are equivalent.

(a) $X$ has the weak $\lambda-B A P$.

(b) There exists an extension operator

$$
\begin{aligned}
& \quad \Phi \in \overline{X \otimes X^{*}} w^{*} \subset \mathcal{L}\left(X^{*}, X^{* * *}\right)=\left(X^{*} \hat{\otimes}_{\pi} X^{* *}\right)^{*} \\
& \text { with }\|\Phi\| \leq \lambda .
\end{aligned}
$$

Proof of Theorem 3.1. (a) $\Rightarrow(\mathrm{b})$. Since the nuclear operators factor through $\ell_{1}$ and the dual space of $\mathcal{N}\left(X, \ell_{1}\right)=X^{*} \hat{\otimes}_{\pi} \ell_{1}$ (because $\ell_{1}$ has the AP) has a simple description, it is reasonable to establish Theorem 3.1(b) first for $Y=\ell_{1}$.

Let $\Phi$ be the extension operator from Theorem 3.3, and let $\left(S_{\nu}\right) \subset \mathcal{F}(X, X)$ be a net such that $S_{\nu}^{*} \rightarrow \Phi$ weak $^{*}$ in $\mathcal{L}\left(X^{*}, X^{* * *}\right)=\left(X^{*} \hat{\otimes}_{\pi} X^{* *}\right)^{*}$. Let $T \in \mathcal{N}\left(X, \ell_{1}\right)=$ $X^{*} \hat{\otimes}_{\pi} \ell_{1}$. We may assume without loss of generality that $\|T\|_{\pi}=1$. We need to show that for every compact subset $K$ of $X$ and for every $\varepsilon>0$ the convex subset

$$
C=\{T S: S \in \mathcal{F}(X, X),\|S x-x\| \leq \varepsilon \quad \forall x \in K\}
$$

of $X^{*} \hat{\otimes}_{\pi} \ell_{1}$ intersects the closed ball $B=\left\{u \in X^{*} \hat{\otimes}_{\pi} \ell_{1}:\|u\|_{\pi} \leq \lambda+\varepsilon\right\}$. If this were not the case, then there would exist $A \in \mathcal{L}\left(\ell_{1}, X^{* *}\right)=\left(X^{*} \hat{\otimes}_{\pi} \ell_{1}\right)^{*}$ with $\|A\|=1$ such that

$$
\begin{aligned}
\lambda+\varepsilon & =\sup \{\operatorname{Re}\langle A, u\rangle: u \in B\} \leq \inf \{\operatorname{Re}\langle A, T S\rangle: T S \in C\} \\
& \leq \lim _{\nu}\left|\left\langle A, T S_{\nu}\right\rangle\right|=\lim _{\nu}\left|\left\langle S_{\nu}^{*}, A T\right\rangle\right|=|\langle\Phi, A T\rangle| \leq \lambda\|A T\|_{\pi} \leq \lambda
\end{aligned}
$$

a contradiction (above, $A T \in X^{*} \hat{\otimes}_{\pi} X^{* *}$ is defined in the usual way: if $T=$ $\sum_{n} x_{n}^{*} \otimes u_{n}$, with $x_{n}^{*} \in X^{*}, u_{n} \in \ell_{1}$, then $\left.A T=\sum_{n} x_{n}^{*} \otimes A u_{n}\right)$. This establishes Theorem 3.1(b) for $Y=\ell_{1}$. 
Let now $Y$ be a Banach space, let $T \in \mathcal{N}(X, Y)$, and let $\varepsilon>0$. By [5, p. 170], there exist $R \in \mathcal{L}\left(\ell_{1}, Y\right)$ and $\hat{T} \in \mathcal{N}\left(X, \ell_{1}\right)$ with $\|R\| \leq 1$ and $\|\hat{T}\|_{\mathcal{N}} \leq\|T\|_{\mathcal{N}}+\varepsilon / \lambda$ such that $T=R \hat{T}$. Let $\left(S_{\alpha}\right) \subset \mathcal{F}(X, X)$ be a net such that $S_{\alpha} \rightarrow I_{X}$ uniformly on compact sets and $\lim \sup _{\alpha}\left\|\hat{T} S_{\alpha}\right\|_{\mathcal{N}} \leq \lambda\|\hat{T}\|_{\mathcal{N}}$. Then

$$
\limsup \left\|T S_{\alpha}\right\|_{\mathcal{N}}=\limsup \left\|R \hat{T} S_{\alpha}\right\|_{\mathcal{N}} \leq \limsup \left\|\hat{T} S_{\alpha}\right\|_{\mathcal{N}}<\lambda\|T\|_{\mathcal{N}}+\varepsilon
$$

By letting $\varepsilon \rightarrow 0$, Theorem 3.1(b) follows.

(b) $\Rightarrow$ (a). Let $T \in \mathcal{F}(X, X)$. Choose $A$ and $V$ as in Lemma 2.4. Then $V^{*}=A T^{*}$ and

$$
\|T\|_{\pi} \leq \limsup _{\alpha}\left\|j_{X} V S_{\alpha}\right\|_{\pi}
$$

for every net $\left(S_{\alpha}\right) \subset \mathcal{F}(X, X)$ converging pointwise to $I_{X}$. Since $j_{X} V \in \mathcal{N}\left(X, X^{* *}\right)$, for every $\varepsilon>0$, we can write

$$
j_{X} V=\sum_{n=1}^{\infty} x_{n}^{*} \otimes x_{n}^{* *}, \quad x_{n}^{*} \in X^{*}, x_{n}^{* *} \in X^{* *},
$$

with $\sum_{n=1}^{\infty}\left\|x_{n}^{*}\right\|\left\|x_{n}^{* *}\right\|<\left\|j_{X} V\right\|_{\mathcal{N}}+\varepsilon / \lambda$.

Now choose an $\ell_{1}(\Gamma)$-space such that $X$ is its quotient space, and denote $q$ : $\ell_{1}(\Gamma) \rightarrow X$ the quotient mapping. Since $q^{*}$ is an isometric embedding, for all $x_{n}^{* *}$, there exist $u_{n}^{* *} \in \ell_{1}(\Gamma)^{* *}$ such that $q^{* *} u_{n}^{* *}=x_{n}^{* *}$ and $\left\|u_{n}^{* *}\right\|=\left\|x_{n}^{* *}\right\|$. Define

$$
U=\sum_{n=1}^{\infty} x_{n}^{*} \otimes u_{n}^{* *} \in \mathcal{N}\left(X, \ell_{1}(\Gamma)^{* *}\right),
$$

and choose a net $\left(S_{\alpha}\right) \subset \mathcal{F}(X, X)$ converging pointwise to $I_{X}$ such that

$$
\begin{aligned}
\limsup _{\alpha}\left\|U S_{\alpha}\right\|_{\mathcal{N}} & \leq \lambda\|U\|_{\mathcal{N}} \leq \lambda \sum_{n=1}^{\infty}\left\|x_{n}^{*}\right\|\left\|u_{n}^{* *}\right\| \\
& =\lambda \sum_{n=1}^{\infty}\left\|x_{n}^{*}\right\|\left\|x_{n}^{* *}\right\|<\lambda\left\|j_{X} V\right\|_{\mathcal{N}}+\varepsilon .
\end{aligned}
$$

Moreover,

$$
\left\|j_{X} V\right\|_{\mathcal{N}}=\left\|V^{*}\right\|_{\mathcal{N}}=\left\|A T^{*}\right\|_{\mathcal{N}} \leq\left\|T^{*}\right\|_{\mathcal{N}}=\left\|j_{X} T\right\|_{\mathcal{N}} .
$$

On the other hand, it can be easily verified that $j_{X} V=q^{* *} U$. Hence, $j_{X} V S_{\alpha}=$ $q^{* *} U S_{\alpha}$. But $U S_{\alpha} \in \mathcal{N}\left(X, \ell_{1}(\Gamma)^{* *}\right)=X^{*} \hat{\otimes}_{\pi} \ell_{1}(\Gamma)^{* *}$ because $\ell_{1}(\Gamma)^{* *}$ has the AP. Hence, $q^{* *} U S_{\alpha}=\left(I_{X^{*}} \otimes q^{* *}\right)\left(U S_{\alpha}\right)$, where $I_{X^{*}} \otimes q^{* *}: X^{*} \hat{\otimes}_{\pi} \ell_{1}(\Gamma)^{* *} \rightarrow X^{*} \hat{\otimes}_{\pi} X^{* *}$. Therefore,

$$
\left\|j_{X} V S_{\alpha}\right\|_{\pi}=\left\|q^{* *} U S_{\alpha}\right\|_{\pi} \leq\left\|U S_{\alpha}\right\|_{\pi}=\left\|U S_{\alpha}\right\|_{\mathcal{N}} .
$$

In conclusion,

$$
\|T\|_{\pi} \leq \limsup _{\alpha}\left\|j_{X} V S_{\alpha}\right\|_{\pi} \leq \limsup _{\alpha}\left\|U S_{\alpha}\right\|_{\mathcal{N}}<\lambda\left\|j_{X} T\right\|_{\mathcal{N}}+\varepsilon .
$$

By letting $\varepsilon \rightarrow 0$, we see that condition (b) of Theorem 3.2 holds. This means that $X$ has the weak BAP.

Corollary 3.4 (see [17]). The weak $\lambda-B A P$ and the $\lambda$-BAP are equivalent for a Banach space $X$ whenever $X^{*}$ has the Radon-Nikodým property. 
Proof. It is well known that if $X^{*}$ has the Radon-Nikodým property, then $\mathcal{S I}(X, Y)$ $=\mathcal{N}(X, Y)$ (with equality of norms) for all Banach spaces $Y$. This makes conditions (b) of Theorems 2.1 and 3.1 identical. So the claim is immediate from the theorems.

\section{The ROLE OF $\ell_{1}$}

Let $X$ be a Banach space. It is well known that $\mathcal{N}\left(X, \ell_{1}\right)=\mathcal{I}\left(X, \ell_{1}\right)$ with equality of norms (since $\ell_{1}$ has the Radon-Nikodým property). Therefore, by Theorem 3.1 and its proof, we immediately get the following reformulation of the weak BAP.

Proposition 4.1. Let $X$ be a Banach space, and let $1 \leq \lambda<\infty$. The following statements are equivalent.

(a) $X$ has the weak $\lambda-B A P$.

(b) For every $T \in \mathcal{I}\left(X, \ell_{1}\right)$ there exists a net $\left(S_{\alpha}\right) \subset \mathcal{F}(X, X)$ such that $S_{\alpha} \rightarrow$ $I_{X}$ pointwise and $\lim \sup _{\alpha}\left\|T S_{\alpha}\right\|_{\mathcal{I}} \leq \lambda\|T\|_{\mathcal{I}}$.

We are going to develop further the proof of Theorem 2.1, including the proof of Lemma 2.4 to show that if $\ell_{1}$ is replaced by $\ell_{1}^{* *}$ in (b) of Proposition 4.1, then this will be a reformulation of the BAP. Thus, using the space $\ell_{1}$, both the weak BAP and the BAP can be characterized via integral operators in an almost identical way.

Proposition 4.2. Let $X$ be a Banach space, and let $1 \leq \lambda<\infty$. The following statements are equivalent.

(a) $X$ has the $\lambda$-BAP.

(b) For every $T \in \mathcal{I}\left(X, \ell_{1}^{* *}\right)$ there exists a net $\left(S_{\alpha}\right) \subset \mathcal{F}(X, X)$ such that $S_{\alpha} \rightarrow$ $I_{X}$ pointwise and $\lim \sup _{\alpha}\left\|T S_{\alpha}\right\|_{\mathcal{I}} \leq \lambda\|T\|_{\mathcal{I}}$.

The proof of Proposition 4.2 relies on the following reformulation of the BAP in terms of separable ideals. According to the terminology of [6], let us say that a closed subspace $Z$ of $X$ is an ideal whenever there exists an extension operator $\Phi \in$ $\mathcal{L}\left(Z^{*}, X^{*}\right)$ with $\|\Phi\|=1$ (i.e., $\Phi$ is a norm-preserving or Hahn-Banach extension operator).

Proposition 4.3. Let $X$ be a Banach space, and let $1 \leq \lambda<\infty$. The following statements are equivalent.

(a) $X$ has the $\lambda$-BAP.

(b) $\|T\|_{\pi} \leq \lambda\|T\|_{\mathcal{I}(Z, Z)}$ for every separable ideal $Z$ in $X$ and every $T \in \mathcal{F}(Z, Z)$.

(c) $\|S\|_{\pi} \leq \lambda\|S\|_{\mathcal{I}(Z, X)}$ for every separable ideal $Z$ in $X$ and every $S \in \mathcal{F}(Z, X)$.

Proof. By Grothendieck's classics (see, e.g., [3, p. 193] or [22, p. 80]), (a) $\Rightarrow$ (c) holds for every Banach space $Z$ (not just for ideals).

(c) $\Rightarrow($ b). Let $j: Z \rightarrow X$ denote the identity embedding. Since $Z$ is an ideal in $X, Z^{*} \hat{\otimes}_{\pi} Z$ is a closed subspace of $Z^{*} \hat{\otimes}_{\pi} X$ (under the natural inclusion $I_{Z^{*}} \otimes j$ : $Z^{*} \hat{\otimes}_{\pi} Z \rightarrow Z^{*} \hat{\otimes}_{\pi} X$ ) (see [21] or [15, Theorem 3.4], where the converse is also proven). Hence, for all $T \in \mathcal{F}(Z, Z)$,

$$
\|T\|_{\pi}=\|j T\|_{\pi} \leq \lambda\|j T\|_{\mathcal{I}} \leq \lambda\|T\|_{\mathcal{I}} .
$$

$(b) \Rightarrow(a)$. From $[\underline{8}$ or $[23$ we know that every separable subspace is contained in a separable ideal. Therefore, by Theorem 2.2, every separable subspace of $X$ is contained in a separable closed subspace $Y$ of $X$ having the $\lambda$-BAP. By a result due to Johnson [9] (see, e.g., [2, Theorem 9.7]), this means that $X$ has the $\lambda$-BAP. 
Proof of Proposition 4.2. (a) $\Rightarrow$ (b) is obvious as noted already in the Introduction.

(b) $\Rightarrow(\mathrm{a})$. Let $Z$ be a separable ideal in $X$. Then $Z$ is a quotient of $\ell_{1}$ (as all separable Banach spaces are). As in the beginning of the proof of Theorem 2.1. applying Lemma 2.3, we get from (b) that for every $U \in \mathcal{I}\left(X, Z^{* *}\right)$ there exists a net $\left(S_{\alpha}\right) \subset \mathcal{F}(X, X)$ such that $S_{\alpha} \rightarrow I_{X}$ pointwise and

$$
\lim \sup _{\alpha}\left\|U S_{\alpha}\right\|_{\pi} \leq \lambda\|U\|_{\mathcal{I}}
$$

Let $\Phi \in \mathcal{L}\left(Z^{*}, X^{*}\right)$ be a norm-preserving extension operator. Consider

$$
T=\sum_{n=1}^{m} z_{n}^{*} \otimes z_{n} \in \mathcal{F}(Z, Z)=Z^{*} \otimes Z .
$$

As in the proof of Lemma 2.4 we find $A \in \mathcal{L}\left(Z^{*}, Z^{*}\right)$ with $\|A\|=1$ such that

$$
\|T\|_{\pi}=\sum_{n=1}^{m}\left(A z_{n}^{*}\right)\left(z_{n}\right)=\sum_{n=1}^{n}\left(\Phi A z_{n}^{*}\right)\left(z_{n}\right)=\operatorname{trace}(V),
$$

where

$$
V=\sum_{n=1}^{m} \Phi A z_{n}^{*} \otimes z_{n} \in \mathcal{F}(X, Z) .
$$

It is easily verified that $V^{*}=\Phi A T^{*}$.

For $j_{Z} V \in \mathcal{I}\left(X, Z^{* *}\right)$, choose a net $\left(S_{\alpha}\right) \subset \mathcal{F}(X, X)$ converging pointwise to $I_{X}$ such that

$$
\underset{\alpha}{\limsup }\left\|j_{Z} V S_{\alpha}\right\|_{\pi} \leq \lambda\left\|j_{Z} V\right\|_{\mathcal{I}}
$$

But

$$
\left\|j_{Z} V\right\|_{\mathcal{I}}=\left\|V^{*}\right\|_{\mathcal{I}}=\left\|\Phi A T^{*}\right\|_{\mathcal{I}} \leq\left\|T^{*}\right\|_{\mathcal{I}}=\|T\|_{\mathcal{I}} .
$$

On the other hand, similar to the proof of Lemma 2.4.

$$
\|T\|_{\pi}=\lim _{\alpha} \operatorname{trace}\left(V S_{\alpha} j\right) \leq \limsup _{\alpha}\left\|V S_{\alpha} j\right\|_{\pi},
$$

where $j: Z \rightarrow X$ is the identity embedding. Since $V S_{\alpha} j=\left(j^{*} \otimes I_{Z}\right)\left(V S_{\alpha}\right)$ (with respect to $\left.j^{*} \otimes I_{Z}: X^{*} \otimes Z \rightarrow Z^{*} \otimes Z\right)$ and $X^{*} \otimes_{\pi} Z$ is a subspace of $X^{*} \otimes_{\pi} Z^{* *}$, we have

In conclusion,

$$
\left\|V S_{\alpha} j\right\|_{\pi} \leq\left\|V S_{\alpha}\right\|_{\pi}=\left\|j_{Z} V S_{\alpha}\right\|_{\pi}
$$

$$
\|T\|_{\pi} \leq \lambda\|T\|_{\mathcal{I}}
$$

which means, according to Proposition 4.3, that $X$ has the $\lambda$-BAP.

For a Banach operator ideal $\mathcal{A}$, let us denote by $\mathcal{A}^{*}$ the dual operator ideal of $\mathcal{A}$. Its components are $\mathcal{A}^{*}(X, Y)=\left\{T \in \mathcal{L}(X, Y): T^{*} \in \mathcal{A}\left(Y^{*}, X^{*}\right)\right\}$ with $\|T\|_{\mathcal{A}^{*}}=\left\|T^{*}\right\|_{\mathcal{A}}$. (The notation $\mathcal{A}^{*}$ means adjoint ideal in [4] and [20], where the dual operator ideal is denoted by $\mathcal{A}^{d}$ and $\mathcal{A}^{\text {dual }}$, respectively.)

Let $\mathcal{P}$ denote the ideal of absolutely summing operators (1-summing in [4]). It is known that $\mathcal{P}^{*}=\mathcal{D}_{\infty}$, the ideal of $\infty$-dominated operators (see, e.g., [20, 17.4]). It follows that the BAP is also the same as the BAP for $\mathcal{D}_{\infty}$.

Corollary 4.4. Let $X$ be a Banach space, and let $1 \leq \lambda<\infty$. The following statements are equivalent.

(a) $X$ has the $\lambda$-BAP.

(b) $X$ has the $\lambda$-BAP for $\mathcal{D}_{\infty}$. 
Proof. By Proposition 4.2. we only need to verify that $\mathcal{I}\left(X, \ell_{1}^{* *}\right)=\mathcal{D}_{\infty}\left(X, \ell_{1}^{* *}\right)$ with the equality of norms. It is well known that $\mathcal{I} \subset \mathcal{P}$ and, therefore, $\mathcal{I}=$ $\mathcal{I}^{*} \subset \mathcal{P}^{*}=\mathcal{D}_{\infty}$. Hence, $\mathcal{I}\left(X, \ell_{1}^{* *}\right) \subset \mathcal{D}_{\infty}\left(X, \ell_{1}^{* *}\right)$ and $\|T\|_{\mathcal{D}_{\infty}} \leq\|T\|_{\mathcal{I}}$ for all $T \in \mathcal{I}\left(X, \ell_{1}^{* *}\right)$. On the other hand, let $T \in \mathcal{P}^{*}\left(X, \ell_{1}^{* *}\right)$, i.e., $T^{*} \in \mathcal{P}\left(\ell_{\infty}^{* *}, X^{*}\right)$. It is known that $\mathcal{P}\left(\ell_{\infty}, X^{*}\right)=\mathcal{I}\left(\ell_{\infty}, X^{*}\right)$ with equal norms (see, e.g., 44, p. 99]). Therefore, $\left.T^{*}\right|_{\ell_{\infty}} \in \mathcal{I}\left(\ell_{\infty}, X^{*}\right)$ and $T=\left.\left(\left.T^{*}\right|_{\ell_{\infty}}\right)^{*}\right|_{X} \in \mathcal{I}\left(X, \ell_{1}^{* *}\right)$. Moreover,

$$
\|T\|_{\mathcal{I}} \leq\left\|\left(\left.T^{*}\right|_{\ell_{\infty}}\right)^{*}\right\|_{\mathcal{I}}=\left\|\left.T^{*}\right|_{\ell_{\infty}}\right\|_{\mathcal{I}}=\left\|\left.T^{*}\right|_{\ell_{\infty}}\right\|_{\mathcal{P}} \leq\left\|T^{*}\right\|_{\mathcal{P}}=\|T\|_{\mathcal{D}_{\infty}}
$$

as needed.

Let us conclude with the remark that in the metric case (i.e., when $\lambda=1$ ), we have alternative proofs of Theorems 2.1 and 3.1 and of Proposition 4.2, which rely on results from [1, 13, [10, such as, for instance, $X$ has the metric AP if and only if $\mathcal{F}(Y, X)$ is an ideal in $\mathcal{L}(Y, X)$ for every separable Banach space $Y$ (see [13, Theorem 1.1]).

\section{OPEN PROBLEMS}

The inclusion $\mathcal{A} \subset \mathcal{B}$ for Banach operator ideals $\mathcal{A}$ and $\mathcal{B}$ (recalled in the Introduction) provides a partial ordering on the class of all Banach operator ideals with $\mathcal{N}$ as the smallest and $\mathcal{L}$ as the largest elements. Through the notion of the BAP for $\mathcal{A}$, every Banach operator ideal $\mathcal{A}$ yields some kind of AP. In particular, let us say that $\mathcal{A}$ yields the $B A P$ if the $\lambda$-BAP coincides with the $\lambda$-BAP for $\mathcal{A}$; and it is similar in the case of the weak BAP. With the ample choice of different Banach operator ideals, many obvious natural questions arise. For instance, we know that $\mathcal{W}$ yields the weak BAP.

Problem 5.1. Are there larger Banach operator ideals than $\mathcal{W}$ yielding the weak BAP? Or is $\mathcal{W}$ a maximal element here?

To be more specific, one may ask the following.

Problem 5.2. Does the ideal $\mathcal{R N}$ of Radon-Nikodým operators (see, e.g., 20, p. 337]) yield the weak BAP? Does $\mathcal{R N}^{*}$ yield the weak BAP?

If $\mathcal{R N}^{*}$ yielded the weak BAP, then one would immediately have the known result (see Corollary 3.4) that the weak BAP and the BAP are equivalent for $X$ whenever $X^{*}$ has the Radon-Nikodým property.

Recall that $\mathcal{W}, \mathcal{R N}$, and (hence also) $\mathcal{R N}^{*}$ are so-called classical Banach operator ideals (in the terminology of 4) or closed operator ideals (in the terminology of [20]). That is, their operator ideal norm is the usual operator norm. Since $\mathcal{W}$ yields the weak BAP and $\mathcal{L}$ yields the BAP, one may ask as follows.

Problem 5.3. Is there the largest of the classical Banach operator ideals yielding the weak BAP? Is there the smallest of the classical Banach operator ideals yielding the BAP?

We know that $\mathcal{N} \subset \mathcal{I} \subset \mathcal{W} \subset \mathcal{L}$. We also know that $\mathcal{N}$ yields the weak $\mathrm{BAP}, \mathcal{I}$ yields the BAP, $\mathcal{W}$ yields again the weak BAP, and $\mathcal{L}$ yields again the BAP. But what is happening between $\mathcal{I}$ and $\mathcal{W}$ ? There are, for instance, $\mathcal{P}$ and $\mathcal{P}^{*}=\mathcal{D}_{\infty}$ between them. We saw that $\mathcal{P}^{*}$ yields the BAP.

Problem 5.4. Does $\mathcal{P}$ yield the BAP? 
We conclude with a less obvious question.

Problem 5.5. Let $\mathcal{A}$ be an arbitrary Banach operator ideal. Could the $\lambda$-BAP for $\mathcal{A}$ of a Banach space $X$ be defined equivalently by the following (at least formally) weaker condition: For every Banach space $Y$ and for every operator $T \in \mathcal{A}(X, Y)$ there exists a net $\left(T_{\alpha}\right) \subset \mathcal{F}(X, Y)$ such that $T_{\alpha} \rightarrow T$ pointwise and

$$
\limsup \left\|T_{\alpha}\right\|_{\mathcal{A}} \leq \lambda\|T\|_{\mathcal{A}} ?
$$

The answer is obviously "yes" if $\mathcal{A}=\mathcal{L}$, and also if $\mathcal{A}=\mathcal{W}$ (see 18, Theorem 3.6]).

Finally, let the authors admit that the original objective of their work was to approach the famous long-standing open problem (see, e.g., [2, Problem 3.8]). Is the AP of $X^{*}$ always the metric AP (i.e., the 1-BAP)? This problem was reformulated in different ways using the weak BAP in [11.

\section{ACKNOWLEDGEMENT}

The authors thank the referee for helpful suggestions.

\section{REFERENCES}

[1] T.A. Abrahamsen, V. Lima, and Å. Lima. Unconditional ideals of finite rank operators. II. Houston J. Math. 35 (2009) 627-646.

[2] P.G. CasazzA. Approximation properties. In: W.B. Johnson and J. Lindenstrauss (eds.), Handbook of the Geometry of Banach Spaces. Volume 1, Elsevier (2001) 271-316. MR.1863695 (2003f:46012)

[3] A. Defant AND K. Floret. Tensor Norms and Operator Ideals. North-Holland Mathematics Studies 176 (1993). MR.1209438 (94e:46130)

[4] J. Diestel, H. Jarchow, And A. Tonge. Absolutely Summing Operators. Cambridge University Press, Cambridge Studies in Advanced Mathematics 43 (1995). MR1342297 (96i:46001)

[5] J. Diestel And J.J. Uhl, JR. Vector Measures. Mathematical Surveys 15, Amer. Math. Soc., Providence, Rhode Island (1977). MR0453964 (56:12216)

[6] G. Godefroy, N.J. Kalton, And P.D. Saphar. Unconditional ideals in Banach spaces. Studia Math. 104 (1993) 13-59. MR.1208038 (94k:46024)

[7] A. Grothendeck. Produits tensoriels topologiques et espaces nucléaires. Mem. Amer. Math. Soc. 16 (1955). MR0075539 (17:763c)

[8] S. Heinrich AND P. MANKIEWICZ. Applications of ultrapowers to the uniform and Lipschitz classification of Banach spaces. Studia Math. 73 (1982) 225-251. MR675426 (84h:46026)

[9] W.B. Johnson. A complementary universal conjugate Banach space and its relation to the approximation problem. Israel J. Math. 13 (1972) 301-310. MR0326356 (48:4700)

[10] Å. LIMA AND E. OJA. Ideals of finite rank operators, intersection properties of balls, and the approximation property. Studia Math. 133 (1999) 175-186. MR.1686696 (2000c:46026)

[11] Å. LimA And E. OJA. The weak metric approximation property. Math. Ann. 333 (2005) 471-484. MR2198796 (2006i:46025)

[12] V. LimA. The weak metric approximation property and ideals of operators. J. Math. Anal. Appl. 334 (2007) 593-603. MR2332578 (2008g:46022)

[13] V. Lima AND Å. Lima. Ideals of operators and the metric approximation property. J. Funct. Anal. 210 (2004) 148-170. MR2052117 (2004m:46047)

[14] A. Lissitsin, K. Mikkor, And E. OJA. Approximation properties defined by spaces of operators and approximability in operator topologies. Illinois J. Math. 52 (2008).

[15] E. OJA. Operators that are nuclear whenever they are nuclear for a larger range space. Proc. Edinburgh Math. Soc. 47 (2004) 679-694. MR2097268 (2005g:46040)

[16] E. OJA. Lifting bounded approximation properties from Banach spaces to their dual spaces. J. Math. Anal. Appl. 323 (2006) 666-679. MR2262236 (2007k:46025)

[17] E. OJA. The impact of the Radon-Nikodým property on the weak bounded approximation property. Rev. R. Acad. Cien. Serie A. Mat. 100 (2006) 325-331. MR2267414 (2007h:46027) 
[18] E. OJA. The strong approximation property. J. Math. Anal. Appl. 338 (2008) 407-415. MR2386425

[19] E. OJA. Inner and outer inequalities with applications to approximation properties. Preprint (2008).

[20] A. Pietsch. Operator Ideals. North-Holland Publishing Company, Amsterdam-New YorkOxford (1980). MR582655 (81j:47001)

[21] N. RandrianantoAnina. Complemented copies of $\ell^{1}$ and Petczyński's property $\left(V^{*}\right)$ in Bochner function spaces. Canad. J. Math. 48 (1996) 625-640. MR1402332 (97j:46035)

[22] R.A. RyAN. Introduction to Tensor Products of Banach Spaces. Springer Monographs in Mathematics, Springer-Verlag, London (2002). MR1888309 (2003f:46030)

[23] B. Sims And D. Yost. Linear Hahn-Banach extension operators. Proc. Edinburgh Math. Soc. 32 (1989) 53-57. MR.981992 (90b:46042)

Department of Mathematics, University of Agder, Serviceboks 422, 4604 KristianSAND, NORWAY

E-mail address: Asvald.Lima@uia.no

Department of Mathematics, University of Missouri-Columbia, Columbia, Missouri 65211

E-mail address: lima@math.missouri.edu

Current address: Aalesund University College, Service Box 17, N-6025 Ålesund, Norway

E-mail address: Vegard.Lima@gmail.com

Faculty of Mathematics and Computer Science, University of Tartu, J. Liivi 2, EE-50409 TARTU, Estonia

E-mail address: eve.oja@ut.ee 